\title{
Pratiques
}

Linguistique, littérature, didactique

$157-158 \mid 2013$

Théories et pratiques des genres

\section{Un genre de discours miniature : pour un modèle de l'anecdote}

Karine Abiven

\section{(2) OpenEdition}

Journals

Édition électronique

URL : http://journals.openedition.org/pratiques/3744

DOI : 10.4000 /pratiques.3744

ISSN : 2425-2042

Éditeur

Centre de recherche sur les médiations (CREM)

Édition imprimée

Date de publication : 1 juin 2013

Pagination : 119-132

\section{Référence électronique}

Karine Abiven, « Un genre de discours miniature : pour un modèle de l'anecdote », Pratiques [En ligne], 157-158 | 2013, mis en ligne le 18 décembre 2017, consulté le 19 avril 2019. URL : http:// journals.openedition.org/pratiques/3744; DOI : 10.4000/pratiques.3744

(c) Tous droits réservés 


\title{
Un genre de discours miniature : pour un modèle de l'anecdote
}

\author{
Karine Abiven \\ EA 4509 « Sens Texte Informatique Histoire », \\ Université Paris-Sorbonne
}

Un Domestique effrayé avertit un jour Budé dans son cabinet, que le feu venoit de prendre à la maison : avertissez ma femme, répondit-il froidement; vous savez que je ne me mêle point du ménage.

Abbé de Raynal, Anecdotes littéraires, Paris, Durand, 1750, p. 2-3.

L'anecdote est un genre de récit courant qui n'a pas souvent retenu l'attention des chercheurs ${ }^{(1)}$, peut-être en raison de son caractère fruste et du discrédit dont elle fait l'objet (pensons aux précautions énonciatives qui l'escortent souvent: «ce n'est qu'anecdotique », "c'est seulement pour l'anecdote »). À étudier ses propriétés formelles et ses usages discursifs, l'anecdote apparaît pourtant comme un dispositif qui permet de mieux connaître de nombreuses fonctions du récit : l'exemplarité morale ou l'usage pédagogique des exemples, les processus inductifs ou probatoires dans l'argumentation, la digression au sein d'un plus ample récit, etc. Il a semblé particulièrement fécond d'aborder cette question sur un corpus de textes de la fin de la première modernité, pour des raisons à la fois lexicales et historiques. Le mot anecdote apparaît en effet dans les dictionnaires de langue française (puis dans ceux des autres langues européennes) dans la période qui va des années 1685 au milieu du XVIII siècle. On remarque simultanément une floraison d'ouvrages propres à accueillir ce bref genre de récit : re-

(1) Sauf en linguistique textuelle, avec les travaux de J.-M. Adam, Genres de récits : narrativité et généricité des textes, Louvain-la-Neuve/Paris, Academia/L'Harmattan, coll. "Sciences du langage, carrefours et points de vue », $\mathrm{n}^{\circ} 4,2011$, et avec G. Lugrin, «Variations des ancrages énonciatifs et fictionnalisation d'une anecdote d'Albert Camus », dans G. Philippe (dir.), L'Ancrage énonciatif des récits de fiction, Langue Française, vol. 128, ${ }^{\circ} 1$, déc. 2000, p. 96-112, ainsi que de V. Magri-Mourgues, "Hétérogénéité, cohésion et cohérence : le statut de l'anecdote digressive dans un récit de voyage ", dans A. Jaubert (dir.), Cohésion et cohérence. Études de linguistique textuelle, Paris, Éd. de 1'ENS, 2005, p. 157173. 
cueils d'anecdotes (nommés ana), Mémoires de cour, Vies d'écrivains, etc. Il commence aussi à l'époque à faire l'objet d'un métadiscours qui perdure aujourd'hui, où l'on met en doute sa véridicité tout en manifestant une curiosité et un désir de raconter. L'essor de l'anecdote et du discours qui l'entoure, de la fin du XVII ${ }^{\mathrm{e}}$ siècle au mitan du siècle suivant, laisse penser que se cristallise alors une forme de micro-récit spécifique.

Selon une définition intuitive du mot, l'anecdote est cette vignette narrative qu'on lance dans une conversation, qu'on épingle en manchette d'un journal ou qu'on lit dans une biographie. C'est un récit minimal, caractérisé par une prétention à la véridicité et visant à susciter un affect chez le récepteur. Même assez restrictive, cette définition recouvre un nombre de textes virtuellement infini, parmi lesquels on se contentera ici de glaner, en prélevant des échantillons dans des textes hétérogènes. Deux exemples peuvent illustrer le type de séquences en question. Tallemant des Réaux, un auteur des salons mondains du XVII siècle, nous procure un réservoir idéal d'exemples : dans les années 1657-1659, il collecte des centaines d'anecdotes au sujet des rois, des écrivains et de toutes sortes de personnages connus ou inconnus aujourd'hui, dans un épais manuscrit intitulé Historiettes. Il conte ainsi en une petite touche narrative un détail du règne d'Henri IV :

Il est bien certain que le Roy dit, un jour que Conchine, depuis mareschal d'Ancre, l'estoit allé saluer à Monceaux : «Si j'estois mort, cet homme-là ruineroit mon royaume ${ }^{(2)} »$.

On peut confronter ce récit à ceux composés un siècle plus tard par Voltaire, qui consacre quatre chapitre de son célèbre ouvrage historiographique, le Siècle de Louis XIV, à des «particularités et anecdotes »; il écrit ainsi dans une forme concise, au sujet de Louis XIVet de Mazarin :

C'était une anecdote très connue à la cour, qu'il avait dit après la mort du cardinal : «Je ne sais pas ce que j'aurais fait, s'il avait vécu plus longtemps ${ }^{(3)}$ ».

Chute finale sous forme d'un discours rapporté plus ou moins apocryphe, forme narrative particulièrement ramassée : un air de famille apparaît, du point de vue de la forme et des motifs, qui dessine un horizon générique. Le but est ici de mieux cerner ce genre intuitivement identifiable en cherchant les traits définitoires de la séquence textuelle qui active cette compétence générique.

Le modèle qu'on esquisse ici sert à découper des brèves séquences à l'intérieur de vastes récits : cette méthode, venue de la linguistique textuelle, permet de rendre compte de l'hétérogénéité de textes longs (y compris s'ils apparaissent comme tout uniment narratifs), comme les Mémoires ou les récits de vie. Quels sont donc les marqueurs spécifiques de telles séquences de micro-récits, qui les constituent en genre de discours identifiable?

(2) G. Tallemant des Réaux, Historiettes, éd. A. Adam et G. Delassault, Paris, Gallimard, coll. «Bibliothèque de la Pléiade », 1960-1961, t. I, p. 9.

(3) Voltaire, Le Siècle de Louis XIV [1753], chapitre XXIV « Particularités et anecdotes », éd. J. Hellegouarc'h et S. Menant, Paris, Librairie Générale Française, Livre de Poche, coll. « Bibliothèque classique », 2005, p. 571. 


\section{Un exemplaire représentatif du prototype}

L'anecdote est un récit minimal, au sens assez large de « représentation non contradictoire d'au moins deux événements (ou d'un état et d'un événement) asynchrones ${ }^{(4)}{ }^{\prime}$. Le problème des critères nécessaires et suffisants pour pouvoir parler de récit a souvent été soulevé, que ce soit du point de vue qualitatif(la qualité de l'événement représenté), ou quantitatif (sa taille textuelle critique ${ }^{(5)}$ ). Sans doute faut-il caractériser l'anecdote plutôt par l'absence de complexité que par le critère de la brièveté, toujours relatif. Sans fixer de longueur standard, on peut supposer qu'une structuration minimale est requise pour que se cristallise un récit. Elle suppose ainsi, comme toute séquence narrative, une succession temporelle, causale et intentionnelle autour d'au moins une action.

Comme forme d'énoncé relativement stable, l'anecdote présente des régularités qu'on peut regrouper par faisceaux. Pour ce faire, la théorie du prototype apparaît comme particulièrement pertinente : inséparable de l'idée d'une échelle de ressemblance par rapport à un modèle, elle permet de penser les occurrences selon des degrés de prototypicité et non pas en termes d'exclusion ou d'appartenance - ce qui convient bien à des objets «flous » tels que les genres. Plus ou moins proches du prototype, les anecdotes sont donc plus ou moins identifiables comme telles, et activent à des degrés divers la compétence générique indissociables des « genres de discours ${ }^{(6)} »$.

Il s'agit donc de définir le prototype de la séquence anecdotique, c'est-à-dire, à proprement parler, de s'appuyer sur le « meilleur exemplaire ${ }^{(7)}$ » de celle-ci - cette notion étant bien sûr proprement heuristique. Certains extraits de recueils d'anecdotes peuvent vraisemblablement faire office d'occurrences modèles : leur configuration sérielle présente l'anecdote déjà isolée de la macrostructure, et comme prédécoupée. Cet extrait du recueil intitulé Menagiana ${ }^{\left({ }^{(}\right)}$ peut servir de point de départ à l'observation :

[P1] Madame Mazarin \& madame la Connétable Colonne ; passant à Arles avec

(4) G. Prince, «Récit minimal et narrativité », dans S. Brédane, Fr. Revaz, M. Viegnes (dir.), Le Récit minimal. Du minime au minimalisme. Littérature, arts, media, Paris, Presses de la Sorbonne nouvelle, 2012, p. 23-32 ; ici p. 24-25.

(5) Pour une synthèse théorique sur «1'empan textuel minimal » de la narrativité, voir Fr. Revaz, Introduction à la narratologie, Bruxelles, De Boeck et Duculot, 2009, p. 82-87.

(6) M. Bakhtine, «Les genres du discours » [1952-1953], Esthétique de la création verbale, Paris, Gallimard, coll. "Bibliothèque des Idées », 1984. Pour une articulation des notions de séquence et de genre de discours, voir J.-M. Adam, Les Textes, types et prototypes. Récit, description, argumentation, explication et dialogue, [Nathan, 1997], $2{ }^{\mathrm{e}}$ édition, Paris, Armand Colin, 2008 [rééd. 2011], p. 12-13. La postérité de ce chapitre de Bakhtine est retracée par S. Moirand dans un développement intitulé « 50 ans après Bakhtine, où en sommes nous? », dans Quelles catégories descriptives pour la mise au jour des genres de discours?, www.icar.univ-lyon2.fr, page consultée le 20 janvier 2013.

(7) C'est la définition standard du prototype, même s'il est souvent compris moins comme «meilleur exemplaire » que comme « combinaison de propriétés typiques » (G. Kleiber, La Sémantique du prototype. Catégories et sens lexical, Paris, P. U. F, 1990, p. 186).

(8) Les ana, ces recueils d'anecdotes et de pensées prêtées à un auteur célèbre en son temps, sont intitulés d'après le patronyme de l'auteur augmenté du suffixe -ana : les paroles de Gilles Ménage sont les Menagiana, les dits de François Charpentier sont les Carpentariana, etc. 
chacune un petit cofre à serrer des pierreries : Madame de Sevigné [P2] qu'elles y allerent voir chez M. de Grignan, [P3] s'appercevant qu'elles étoient en linge sale, [P4] leur envoya le soir à chacune une douzaine de chemises avec une lettre qui commençoit ainsi : [P5] Vous êtes comme des Héroïnes de Roman ; force pierreries, \& point de linge blanc ${ }^{(9)}$.

Dans cette séquence, où l'on a numéroté les propositions susceptibles d'avoir un rôle narratif, se retrouvent les traits prototypiques déjà établis pour le récit en général. On le sait, la définition minimale du récit est d'être la relation d'actions temporellement ordonnées $(t \rightarrow t+n)$ et tendue entre un début et une fin qui nouent et de dénouent une action ${ }^{(10)}$. Ici, cinq propositions permettent de passer du moment où les deux protagonistes sont décrites (à Arles, avec leur coffre à bijoux, en P1) au moment où un autre acteur porteur d'une évaluation sur la situation (Madame de Sévigné s'aperçoit de leur inconvenance [P2 et P3] et le leur signifie par un acte [P4] et une parole spirituelle [P5]). Sur le plan de la textualité, il s'agit bien d'une suite de propositions liées progressant vers une fin. Ces propositions temporellement ordonnées peuvent être réduites à des statuts fonctionnels stables ${ }^{(11)}$. Si l'on reprend la description que donne J.-M. Adam des macropropositions narratives $(\mathrm{Pn})$ prototypiques et de leur fonction ${ }^{(12)}$, on peut découper ainsi la séquence :

Pn1 (= P1) (Situation initiale [orientation]) : « Madame Mazarin \& madame la Connétable Colonne ; passant à Arles avec chacune un petit cofre à serrer des pierreries $\gg$

Pn2 (= P2 et P3) (Complication [déclencheur 1]) : « Madame de Sevigné qu'elles y allerent voir chez M. de Grignan, s'appercevant qu'elles étoient en linge sale »

Pn3 (= P4) (Action) : « leur envoya le soir à chacune une douzaine de chemises avec une lettre qui commençoit ainsi : »

Pn4 (= P5) (Résolution [déclencheur 2]) « Vous êtes comme des Héroïnes de Roman ; force pierreries, \& point de linge blanc»

S'il l'on postule que de semblables séquences constituent un exemplaire re-

(9) Menagiana, sive excerpta ex ore Aegidii Menagi, Paris, F. et P. Delaulne, 1693, p. 96-97.

(10) Le récit est généralement perçu comme « une unité tensive (et même doublement tensive : tension représentante et tension représentée) entre une clôture initiale et une clôture finale » (J. Bres, «À la recherche de la narrativité : éléments pour une théorisation praxématique », dans J. Bres (dir.), Du récit, encore, Cahiers de praxématique, Montpellier, Université Paul Valéry-Montpellier III, n 11, 1988, p. 75-100; ici p. 76).

(11) Voir la théorie du récit ordinaire de W. Labov pour une des premières formalisations de ces fonctions (Le Parler ordinaire. La langue dans les ghettos noirs des Etats-Unis [1972, trad. française, 1978], Paris, Minuit, 1993, Chapitre 9, « La transformation du vécu à travers la syntaxe narrative », p. 457-503).

(12) Le schéma des propositions narratives est le suivant, «Pn » désignant les propositions narratives, et « $\mathrm{m} »$ les moments (rappelé par exemple dans Les Textes, types et prototypes. Récit, description, argumentation, explication et dialogue, op. cit., p. 57, p. 66) :

[Pno Résumé et/ou Entrée-préface]

Pn1 Situation initiale-orientation $(\mathrm{m} 1)$

Pn2 Complication Déclencheur 1 (nœud)

Pn3 Actions ou Évaluation $(\mathrm{m} 2)+(\mathrm{m} 3)+(\mathrm{m} 4)$

Pn4 Résolution Déclencheur 2 (dénouement)

Pn5 Situation finale (m5)

[Pn $\Omega$ Évaluation finale (morale) et/ou Chute]. 
présentatif de la catégorie, quelques constats préliminaires s'imposent concernant le prototype anecdotique. D'abord, l'absence de complexité et la brièveté de la séquence impliquent que les macro-propositions narratives sont actualisées en surface par une seule proposition syntaxique, voire seulement un ou plusieurs constituants phrastiques (comme le nom expansé en P1). Ensuite, 1'anecdote est un récit minimal, et à ce titre toutes les propositions typiques du récit n'y sont sans doute pas nécessaires. L'absence de développement central, en particulier, est vraisemblablement constitutif de ces séquences, toute amplification trop conséquente faisant passer à des genres de récits plus développés, comme la nouvelle. Le début du procès $(\mathrm{Pn} 2)$ et son déroulement $(\mathrm{Pn} 3)$ sont réalisés par une seule proposition syntaxique, ce qui implique que Pn3 est réduite, voire facultative : l'action au centre d'un récit très bref ne comporte pas nécessairement plusieurs moments distincts. Pour des raisons similaires de concision, Pn5 (Situation finale) et $\operatorname{Pn} \Omega$ (Morale) semblent optionnelles, puisque la seule présence de la chute (Pn4) suffit à clore le récit (elle comprend en elle-même la morale implicite de l'histoire, les lecteurs ne manquant pas de déceler le jugement axiologique découlant de cette raillerie). Si le caractère facultatif de la situation finale ne doit pas surprendre (quand le dénouement est net, la situation finale n'est pas nécessaire), l'absence de morale explicite devra être commentée plus bas.

Le prototype de l'anecdote se reconnaît donc par une mise en tension particulièrement économique, entre une ouverture et une clôture marquées. Ce sont les caractéristiques de ce cadre que nous allons préciser dans le reste de ce travail.

\section{Un cadre saillant}

L'encadrement de l'anecdote, visible et stéréotypé, constitue vraisemblablement comme un «index générique ${ }^{(13)}$ » : les faisceaux d'indices présents aux bornes de la séquence $(\operatorname{Pn} 1$ et $\operatorname{Pn} 5 / \operatorname{Pn} \Omega)$ fonctionnent comme une signalétique, au sens où ils impliquent une reconnaissance de l'anecdote dans son identité générique.

\section{1. «Un jour » : le « il était une fois » du récit factuel}

L'anecdote isole un moment unique. Un des signaux de l'ouverture de la séquence est un marqueur temporel dont la forme la plus fréquente est « un jour» :

Un jour, entre chien et loup, dans les rues de Paris, il [M. D’Orléans] fut arresté par des voleurs. «Ah, Messieurs, » dit-il en riant, " Vous ouvrez de bonne heure aujourd'huy ${ }^{(14)} »$.

Des variantes comme " une fois », " une autre fois », « l'autre jour », « un soir », etc. sont également récurrentes. «Un jour» et ses variantes ont la même fonction : on décrira celle-ci moins comme un rôle sémantico-syntaxique de com-

(13) J.-M. Schæffer, Qu'est-ce qu'un genre littéraire?, Paris, Éd. du Seuil, coll. "Poétique », 1989, p. 174.

(14) G. Tallemant des Réaux, Historiettes, op. cit., t. I, p. 354. 
plément circonstanciel, mais plutôt, à la suite des travaux de M. Charolles ${ }^{(15)}$, comme un rôle textuel de cadratif. Ces adverbiaux ${ }^{(16)}$ cadratifs, fréquemment placés en tête de phrase ou du moins en zone préverbale, ont vocation à jouer un rôle de structuration du discours. Leur fonction textuelle domine sur leur valeur circonstancielle, ici très réduite (elle se limite à l'indication de la fréquence singulative du récit ${ }^{(17)}$ ). Cette faible informativité est inversement proportionnelle à leur récurrence.

Si l'adverbial « un jour » n'apporte aucune réelle information circonstancielle, il possède d'autres fonctions, notamment celle de segmentation textuelle ${ }^{(18)}$. En position frontale, le cadratif signale que les contenus propositionnels qui suivent doivent être relativisés à une certaine période de temps; cela lui permet d'indexer des contenus qui dépassent les limites de la phrase. Cette portée transphrastique ${ }^{(19)}$ permet de créer un cadre discursif, qui se confond avec celui de l'anecdote :

Un jour il entra dans sa chambre [il s'agit d'un poète de cour et de ses relations avec la reine] ; elle estoit couchée sur son lict, la jupe relevée ; on luy pouvoit voir les cuisses; car le lict n'estoit que de lacis. «Ah! », dit-elle, « où allez-vous ? »Il nie d'en avoir jamais esté amoureux $[\ldots]^{(20)}$.

La portée du cadratif est nette : le SN « un jour» pose un cadre de récit au passé, et le présent « il nie », en manifestant le retour au discours (par un discours narrativisé au présent, coïncidant avec le repère de l'énonciation), montre que la séquence narrative est close. Le cadratif initial permet de rassembler des blocs ou des unités satisfaisant les critères sémantiques dont il est porteur, en l'occurrence le moment ponctuel du passé de l'épisode anecdotique, coupé du présent.

Les cadratifs induisent donc une rupture par rapport à ce qui précède, mais aussi une tension vers la chute. Contrairement aux connecteurs dont ils se rapprochent par ailleurs par leur position détachée souvent en tête de phrase, ils sont fondamentalement orientés vers l'aval du discours ${ }^{(21)}$. Le syntagme « un jour» ou ses équivalents ouvrent un cadre dont on attend la fermeture. L'analyse comme cadratif met en avant la «directionnalité très forte ${ }^{(22)} »$ de ces ouvertures

(15) M. Charolles, L'Encadrement du discours : univers, champs, domaines et espaces, Cahier de Recherche Linguistique, LANDISCO, URA-CNRS 1035 Université Nancy 2, nº, 1-73, 1997 ; et en collaboration avec M.-P. Péry-Woodley, "Introduction », Les Adverbiaux cadratifs. Langue Française, ${ }^{\circ} 148,2005$, p. 3-8.

(16) On prend alors adverbial en un sens large, celui de complément circonstanciel non argumentatif.

(17) Pour reprendre la distinction genettienne entre itératifet singulatif, le récit singulatif « racont[ant] une fois ce qui s'est passé une fois » (G. Genette, Figures III, Paris, Éd. du Seuil, coll. «Poétique », 1972, p. 146).

(18) Voir M. Charolles et M.-P. Péry-Woodley, «Introduction », Les Adverbiaux cadratifs, op. cit, p. 6.

(19) Sur cette notion de portée des unités textuelles liée à des faits de segmentation, voir l'introduction de M. Charolles au numéro 57 de Pratiques, 1988 p. 3-16 («Les plans d'organisation textuelle : périodes, chaînes, portées et séquences »).

(20) G. Tallemant des Réaux, Historiettes, op. cit., t. I, p. 553, les italiques sont de nous.

(21) Voir M. Charolles, A. Le Draoulec, M.-P. Péry-Woodley et L. Sarda, «Temporal and spatial dimensions of discourse organisation ». Journal of French Language Studies, ${ }^{\circ}{ }^{15}$, p. 203 218, accessible sur le site : http ://w3.univ-tlse2.fr. ; consulté le 2 janvier 2013.

(22) M. Charolles et M.-P. Péry-Woodley, «Introduction», Les Adverbiaux cadratifs, op. cit, p. 7. 
de séquence. Il en va de même dans les occurrences où « un jour » est expansé par des compléments, parfois nombreux et imbriqués, qui servent à poser les circonstances du micro-récit. Il s'agit souvent d'une relative restrictive qui reprend un contenu antérieur, comme dans cette anecdote sur Henri IV :

Un jour qu'il se trouva beaucoup de cheveux blancs : «En vérité », dit-il, « ce sont les harangues que l'on m'a faittes depuis mon avenement à la coronne [sic] qui m'ont fait blanchir comme vous voyez ${ }^{(23)} »$.

L'anecdote est reliée au cotexte antérieur par le pronom « il », sujet du verbe d'une relative qui permet de poser brièvement les éléments nécessaires à la chute. Que ce soit l'adverbial cadratif « un jour», ou ses variantes expansées, les marqueurs de cohésion textuelle à l'entame de l'anecdote sont descendants. Les connecteurs-organisateurs en ouverture d'anecdote constituent tout à la fois des facteurs de discontinuité (temporelle et textuelle) et de directionnalité, des marqueurs sémantiques de fréquence (récit singulatif), et des indicateurs de la portée transphrastique de la séquence.

«Un jour» est le « il était une fois » du micro-récit historique : ces incipit (au sens large) sont clairement liés à la généricité ${ }^{(24)}$. Aussi prototypiques l'une que l'autre, ces formules suffisent à signaler l'entrée dans un récit, à cette différence que la seconde opère une suspension de l'univers de croyance du récepteur, et ouvre un horizon d'attente générique de type fictionnel. L'anecdote n'est pas le conte, et le cadratif " un jour », s'il implique une semblable sollicitation d'un horizon d'attente générique, renvoie plutôt au monde de référence, à un petit fait (prétendument) vrai.

\subsection{Le fin mot de l'histoire : clôture du récit}

La clôture est cruciale pour la structuration de tout récit, en raison de la tension décrite plus haut : de sa résolution dépend la perception qu'on a de son unité et de son identité. À défaut de quoi la réception de la séquence narrative risque l'évaluation qui signe son échec : «Et alors ${ }^{(25)}$ ? », manifestant que la séquence n'a pas été interprétée dans ses étapes constitutives, qu'elle est jugée sans intérêt (sans nœud significatif). La fin, est, à tous les sens du terme, le point essentiel du récit, comme le formule Philippe Hamon : la clausule est « la perception de l'interaction, en un point du texte particulier (celui qui précède immédiatement le blanc maximum du texte) de trois paramètres, fin/finition/finalité ${ }^{(26)}$ ». Cette triple fonction est celle la clôture de l'anecdote, au double sens d'organisation interne et de terminaison du récit. Elle clôt la diégèse minuscule, suscite l'acte perlocutoire $^{(27)}$, et signale la complétude de la séquence. Ce dernier point est structurellement important : il implique un mode de lecture prospectif (perce-

(23) G. Tallemant des Réaux, Historiettes, op. cit., t. I, p. 13.

(24) Sur ce «seuil formulaire d'un univers fictionnel», voir J. Adam et U. Heidmann, Textualité et intertextualité des contes : Perrault, Apulée, La Fontaine, Lhéritier, Paris, Éd. Classiques Garnier, coll. «Lire le XVII ${ }^{\mathrm{e}}$ siècle », $\mathrm{n}^{\circ} 2,2010$, p. 235-248; ici p. 235.

(25) W. Labov évoque cette évaluation qui signale la malfaçon du récit ou de sa réception ( $L e$ Parler ordinaire, op. cit., p. 471).

(26) Ph. Hamon, « Clausules », Poétique, 1975, n² 24, p. 495-526, ici p. 497.

(27) Le rôle de la clausule est d' « accentuer émotivement ou pathétiquement un énoncé » (id.). 
vant la tension, on attend la fin, et surtout, atteignant la fin, on comprend le tout) :

Une personne disant à l'illustre Jérôme Bignon, que Rome étoit le siége de la Foy ; cela est vrai, lui répondit-il, mais cette Foy ressemble à de certaines gens qu'on ne trouve jamais au logis ${ }^{(28)}$.

L'immédiateté de la chute, qui repose sur la remotivation du mot siège dans la catachrèse siège de la foi, donne un tour vif à une opinion, rattachée à un homme illustre qui sert de figure d'autorité. Ce micro-récit est manifestement conçu à partir de sa pointe finale qu'il s'agit d'amener habilement par une rapide mise en scène.

Cette structuration aimantée par la fin suppose sans doute un principe de composition rétrograde, comme si l'anecdote était un petit système conçu pour sa chute, vers laquelle elle converge et qui la structure en retour. Sans doute ce modèle rétrograde fonctionne-t-il particulièrement bien pour les récits clos par un discours rapporté : souvent de type comique, ils présentent une tension propre à susciter le rire, qui vient surdéterminer la tension narrative — on pourrait en dire de même pour le mot historique admirable et mémorable. En outre, le discours rapporté, surtout le discours direct, est plus saillant que le récit d'une action en position finale, parce que, comme l'écrit G. Genette, « le discours inséré dans le récit reste discours et forme une sorte de kyste très facile à reconnaître et à localiser ${ }^{(29)}$ ». Le « kyste » de discours rapporté constitue idéalement le fin mot de l'histoire, au double sens de clôture finale et de clausule pointue.

Ces remarques invitent à caractériser la clôture de l'anecdote, à partir des critères dégagés par $\mathrm{Ph}$. Hamon, d'accentuée etfermante (elle « déclenche une activité mémorielle de rétroaction dans la lecture $\left.{ }^{(30)} »\right)$. Elle est aussi stéréotypée, selon un autre de ses critères : même si son but est d'étonner (visée prise en charge par le signifié), elle est attendue, ritualisée, du point de vue du signifiant. «Le sentiment d'achèvement ${ }^{(31)}$ ", écrit encore Hamon, provient généralement « de la saturation d'un modèle ou d'un code implicite ${ }^{(32)}$ », ici celui de la pointe, de la parole mémorable, ou du petit récit à chute qu'on trouve encore dans le genre de la blague.

\subsection{Appareil démarcatif et cadre interactionnel}

Le contour de l'anecdote, tracé du cadratif à la chute, est surdéterminé par une escorte à la fois métadiscursive et pragmatique : résumé et annonce d'une part, récapitulation et évaluation de l'autre. Cet « appareil démarcatif ${ }^{(33)}$ » de l'énoncé est situé en ces « lieux stratégiques qui constituent les frontières externes ou

(28) Carpentariana, éd. Boscheron, Amsterdam, s. 1., 1741, p. 5.

(29) G. Genette, «Frontières du récit », Communications, n 8, 1966, p. 152-163 ; ici p. 161-162 (repris Figures II, Paris, Éd. du Seuil, coll. « Tel Quel», 1969, p. 49-69).

(30) Ph. Hamon, «Clausules », art. cit., p. 509.

(31) Ibid., p. 499.

(32) Id.

(33) Ph. Hamon, «Texte littéraire et métalangage », Poétique, n³1, 1977, p. 261-284; ici p. 266. 
internes » du texte, des lieux où « tend à se développer et à se localiser un discours métalinguistique, implicite ou explicite, du texte sur lui-même et/ou sur les codes en général ${ }^{(34)} \gg$. Ce cadrage est aussi pragmatique, si l'on suit les propositions de Labov, dont les travaux invitent à voir dans l'entrée-préface Pn0 et l'évaluation finale $\operatorname{Pn} \Omega$ des macro-propositions interactives encadrantes. Ces propositions libres ne sont pas incluses dans l'enchaînement causal et temporel du récit, mais constituent autant d'indications adressées au récepteur pour qu'il perçoive puis qu'il interprète le récit correctement.

\subsection{1. «Voici l'histoire " : effets d'annonce}

C'est bien ce qui se produit d'abord dans l'entrée-préface, qui est constituée d'annonces et/ou de résumés de natures diverses. Le type d'entame le plus fréquent est l'expression métatextuelle, qui, en étiquetant la séquence, joue à la fois le rôle d'identifiant et de démarcateur ( « ceci est une anecdote, et c'est ici qu'elle commence $\gg$ ).

On trouve à ce poste toutes les combinaisons possibles de substantifs (historiette, particularité, etc.) et de caractérisants (petite, extraordinaire, surprenante), par exemple :

Il ne sera peut-estre pas hors de propos de rapporter en cét endroit deux petites historiettes qui marquent bien sa facilité \& son humeur enjoüée ${ }^{(35)}$.

Souvent, comme dans cet extrait de la Vie de monsieur d'Ablancourt, la signalétique oriente la lecture, d'une part vers un horizon d'attente générique (car nommer, c'est identifier, inviter à reconnaître), et ensuite vers une évaluation positive (ici l'annonce sert à justifier la nécessité de ces petits récits, et à prévenir l'éventuelle critique de digression). L'étiquetage générique n'est donc pas une désignation objective, il est déjà une orientation axiologique (« Mais voicy une belle histoire de la demoiselle ${ }^{(36)} \gg$ ) et parfois la suggestion d'un modèle littéraire sous-jacent : «Voici une histoire un peu plus tragique », écrit Tallemant pour annoncer un fait divers stylisé, conforme au genre littéraire alors à la mode des « histoires tragiques ${ }^{(37)}$ ». Cette désignation joue un rôle de segmentation séparant la séquence du cotexte, parfois accentuée par des phénomènes d'emphase syntaxique : «La plus belle aventure qui lui soit arrivée, c'est que $[\ldots]^{(38)}$ ».

Cette dénomination des séquences est un type d'annonce qu'on pourrait dire rhématique, pour reprendre la distinction de Genette s'agissant des sous-titres d'une œuvre ${ }^{(39)}$. Symétriquement, l'annonce du récit peut être thématique,

(34) $I d$.

(35) O. Patru, La Vie de monsieur d'Ablancourt [1681], éditée par R. Zuber dans Les Belles infidèles et la formation du goût classique, Paris, Colin, 1968, p. 419-433; ici p. 432. Les italiques sont de nous.

(36) Tallemant des Réaux, Historiettes, op. cit., t. II, p. 902. Les italiques sont de nous.

(37) Ibid., t. I, p. 216.

(38) Ibid., t. I, p. 661.

(39) G. Genette distingue sous-titres thématiques (qui résument le contenu de l'ouvrage) et rhématiques (qui en désignent le genre) ; voir Seuils, Paris, Éd. du Seuil, coll. "Poétique », 1987, p. 78-85. 
c'est-à-dire consister en un très bref résumé, sous la forme d'un syntagme nominal ou de son équivalent :

Mgr et Mme la duchesse de Bourgogne lui faisaient des espiègleries continuelles. Ils firent mettre un jour des pétards tout du long de l'allée, qui, du château de Marly, va à la perspective, où elle logeait ${ }^{(40)}$.

L'anaphore infidèle est ailleurs remarquable à ce poste, car elle fonctionne comme un indice d'exemplification. Les anaphores pronominales du type : «ses belles actions $[\ldots]$ en rapporter une », ou « voici un exemple », permettent d'effectuer une extraction dans un ensemble, en choisissant un cas jugé représentatif. Dans la Vie de monsieur Boileau-Despréaux, un passage évoque la générosité de Boileau par une action choisie parmi plusieurs exemples de ses libéralités : «Mais il ne s'est pas contenté de faire des libéralités à sa mort [...] : en voici un exemple connu de toute la France ${ }^{(41)} \gg$. Ces faits de langue manifestent en surface l'opération de sélection qui est le principe rhétorique de l'exemplum - choix d'un cas parmi un paradigme d'actions comparables (le préfixe ex dans ex-emplum rappelle cette idée d'extraction).

Outre son rôle démarcatif et anaphorique, l'amorce du récit est souvent un acte de langage ${ }^{(42)}$. Une telle mention de l'acte locutoire est un moyen traditionnel d'entrée en matière, aussi bien à l'échelle du texte qu'à celle de la séquence : «Je veux escrire moy mesme les particularitez de ma vie ${ }^{(43)}$ » précise l'historien Marolles au début de ses Mémoires. Le rôle de l'entrée-préface est bien de prendre en charge la dimension interactive du récit : « le Lecteur ne sera pas fâché que je lui en fasse part ${ }^{(44)}$ », écrit Ancillon, au sujet d'une « occasion curieuse ». L'ancrage déictique est net dans l'un des faits de langue récurrents à ce poste, qui est l'usage du présentatif voici : «Voicy ce que j'ai appris de particulier touchant ce grand homme ${ }^{(45)} \gg$. Le présentatif peut aussi opérer un liage de type logique (en particulier causal) avec le cotexte antérieur, avec des formules du type voici pourquoi (dont l'efficacité pour l'annonce de récits enchâssés ne s'est pas démentie jusqu'au roman réaliste du XIX ${ }^{\mathrm{e}}$ siècle) :

C'est apparemment Fonterailles qui irrita le plus Cinq-Mars contre l'Eminentissime, car il estoit engagé contre le cardinal, et voicy pourquoy ${ }^{(46)}$.

On trouve aussi à ce poste des substantifs séquences du présentatif, au sémantisme souvent proche de cause ou origine :

Voicy l'origine de cette secte qu'on appelle les Janssenistes, et qui fait aujourd'huy tant de bruit. La marquise de Sablé dit un jour à la princesse de Guimené $[\ldots]^{(47)}$.

(40) Saint-Simon, Mémoires, Y. Coirault (éd.), Paris, Gallimard, coll. « Bibliothèque de la Pléiade », 1982-1988, t. II, p. 274. Il s'agit de la princesse d'Harcourt. Nos italiques.

(41) P. Des Maizeaux, Vie de monsieur Boileau-Despréaux, Amsterdam, H. Schelte, 1712, p. 301. Les italiques sont de nous.

(42) W. Labov, Le Parler ordinaire, op. cit., p. 467.

(43) Les Mémoires de Michel de Marolles, Paris, A. de Sommaville, 1656, p. 1.

(44) Ch. Ancillon, Mémoires concernant les vies et les ouvrages de plusieurs modernes célèbres dans la république des lettres, Amsterdam, Wetsteins, 1709, p. 100-102.

(45) G. Tallemant des Réaux, Historiettes, op. cit., t. I, p. 191.

(46) Ibid., t. I, p. 277.

(47) Ibid., t. I, p. 512. 
L'anecdote, c'est bien la petite cause susceptible d'expliquer de grands effets, et ce type d'entrée-préface le souligne explicitement.

Enfin, l'entrée-préface de l'anecdote comprend souvent l'expression de la catégorie du médiatif ${ }^{(48)}$, qui place la séquence dans la dépendance d'une source de savoir (médiation épistémique) ou de perception (médiation perceptuelle). L'anecdote est souvent introduite par un « on m'a dit» ou un «j' ai vu », en tant que récit dépourvu d'origine assignée et toujours en circulation. Les Historiettes de Tallemant des Réaux sont ainsi saturées de verbes relevant de la catégorie du médiatif, qui permettent à l'énonciateur de se figurer comme passeur d'une information : le pronom de rang 1 est ainsi sujet des verbes : « entendre », " ouïr » et sa variante « ouïr conter», « apprendre », « savoir », « tenir de », etc., et complément d'objet second des verbes « apprendre » [《 on m'a appris »], « dire », « conter », etc... Le mémorialiste se présente comme une étape dans la chaîne des discours rapportés : «J'ai sceû d'un de mes amis, qui le tenoit de l'abbé Rivière, que $[\ldots]^{(49)} »$. Dans cet ensemble de faits qui constituent le cadre démarcatif, il faut mentionner les expressions topicalisatrices ${ }^{(50)}$ qui apparaissent parfois à l'entame de la séquence : « $\grave{A}$ ce propos, je ne puis m'empêcher de rapporter un échantillon de sa délicatesse ${ }^{(51)} »$, par exemple. Ainsi, ces divers indices précèdent et surdéterminent le cadre ouvert par « un jour».

\subsection{2. Évaluation et commentaire}

Un ensemble de stratégies, on l'a dit au sujet de la clôture du récit, vise à éviter toute évaluation-sanction négative finale de type : « et alors?». Le bon narrateur parvient à « faire en sorte que, son récit terminé, la seule remarque appropriée soit : "vraiment?" ou tout autre expression apte à souligner le caractère mémorable des événements rapportés ${ }^{(52)}{ }^{\prime}$. L'anecdote, dont la survie dépend de son caractère mémorable, doit a fortiori être conçue pour susciter de telles réactions de la part du coénonciateur. Aussi une proposition finale (1'"évaluation », notée $\operatorname{Pn} \Omega$ ) vient-elle souvent aiguiller la réception du récit. Mais le caractère laconique de l'anecdote interdit sans doute que cette proposition soit une morale trop appuyée ou explicite. De quelle nature est-elle donc?

Cette évaluation finale vise souvent à justifier un récit qui pourrait passer pour une digression. Ainsi de cette conclusion d'une anecdote des Mémoires de Bassompierre, où ce grand militaire de la cour de Louis XIII a conté une de ses amourettes avec une petite servante :

Je vous ai voulu dire cette aventure, bien qu'elle soit de personne de peu : mais elle était si jolie que je l'ai regrettée, et eusse désiré pour beaucoup de la pouvoir revoir ${ }^{(53)}$.

(48) Sur la catégorie du médiatif en français, voir Z. Guentchéva, « Manifestations de la catégorie du médiatif dans les temps du français ", Langue française, ${ }^{\circ} 102,1994$, p. 24-40.

(49) G. Tallemant des Réaux, Historiettes, op. cit., t. I, p. 357.

(50) M. Charolles et M.-P. Péry-Woodley, "Introduction», Les Adverbiaux cadratifs, art. cit, p. 2.

(51) Saint-Simon, Mémoires, op. cit., t. IV, p. 88.

(52) W. Labov, Le Parler ordinaire, op. cit., p. 471.

(53) Mémoires de Bassompierre [1665], extrait édité dans Nouvelles du XVII siècle, R. Picard et J. Lafond (éd.), Bibliothèque de la Pléiade, Paris, Gallimard, 1997, p. 123. 
L'épisode galant dont l'héroïne est une personne du peuple n'a pas sa place dans des Mémoires aristocratiques et militaires : il convient donc de le légitimer par une « évaluation».

La dernière étape d'un récit est souvent un énoncé modalisé, que le classement des tropes connaît sous le nom d'épiphrase, "addition d'un commentaire explicatif, d'une réaction affective, qui s'articulent avec ce qui les précède et qui ne sont donc pas sémantiquement détachables ${ }^{(54)}$ ". Chez Tallemant des Réaux, c'est presque toujours un présentatif qui introduit ce type d'énoncé final : «Voylà le bruit qu'on fit courir, mais ce n'est pas la verité, comme nous dirons en suitte $^{(55)}$ » écrit-il entre un récit relatif à Richelieu, et la correction qu'il y apporte; le présentatif voilà, outre sa fonction anaphorique, enclenche une attitude polémique. En concluant des récits de ridicules par des énoncés comme : «Voyez quel courage ! ${ }^{(56)} »$, Tallemant résume et évalue non plus le récit lui-même mais le comportement de l'actant principal : le narrataire est impliqué dans ce jugement grâce à la polyphonie créée par l'antiphrase. On voit ici clairement le rôle d'orientation argumentative et le caractère interactif des énoncés finaux. Ce sont des «enclaves non narratives », comme l'écrit $\mathrm{Cl}$. Bremond sur les « effusions lyriques, sentences didactiques, méditations philosophiques ${ }^{(57)}$ » insérées dans le récit : elles assurent une transition de discours entre plusieurs récits, ou entre le récit et un encadrement discursif.

Si le commentaire final est le lieu privilégié de l'orientation argumentative ${ }^{(58)}$, il peut aussi servir à relier explicitement le moment $t$ du récit au repère de l'énonciation. Ainsi de cet extrait de l'Abrégé de la vie des peintres de Roger de Piles mettant en scène le peintre Appelle :

Mais s'il disoit son sentiment avec simplicité, il recevoit de la même façon celuy des autres : \& pour en éloigner toute complaisance, il se tenoit caché derriére pour écouter ce qu'on en diroit, dans le dessein d'en profiter. De sorte qu'un Cordonnier passant un jour devant la maison d'Apelle, \& y trouvant un Tableau ainsi éxposé, reprit avec liberté quelque défaut qu'il apperçût à une Sandale, laquelle fut changée incontinent aprés : mais le lendemain repassant par le même endroit, tout glorieux de voir qu'on eut profité de sa critique, censura aussi-tôt une Cuisse où il n'y avoit rien à redire : ce qui obligea Apelle de sortir de derriére sa toile, \& de dire au Cordonnier que son jugement ne passoit pas la Sandale; ce qui passa en Provérbe. Je ne say s'il y a beaucoup d'Apelles aujourd'hui, mais il y a des Cordonniers plus que jamais ${ }^{(59)}$.

La fin est ici soulignée par un dispositif en trois temps : la pointe du discours rapporté attribué à Appelle, la mention de la circulation de la sentence jusqu'à l'actualité des coénonciateurs, puis l'évaluation du narrateur, qui sert à la fois à émettre un jugement axiologique sur l'action du mauvais héros de la fable, et à articuler le récit passé au présent de l'énonciation («aujourd'hui [...] il y a des Cordonniers plus que jamais »). Ce genre d'évaluation que Labov appelle des

(54) C. Fromilhague, Les Figures de style, Paris, Nathan, 1995, p. 101.

(55) G. Tallemant des Réaux, Historiettes, op. cit., t. I, p. 282.

(56) Ibid., t. II, p. 516.

(57) Cl. Bremond, Logiques du récit, Paris, Éd. du Seuil, coll. « Poétique», 1973, p. 322.

(58) J.-M. Adam, Genres de récits. Narrativité et généricité des textes, op. cit., p. 83-86.

(59) R. de Piles, Abrégé de la vie des peintres, Paris, F. Muguet, 1699, p. 125. 
chutes disjonctives « ont pour utilité de combler le vide qui sépare la fin des événements rapportés du moment présent ; elles ramènent le narrateur et son auditeur où ils étaient avant d'entrer dans le récit ${ }^{(60)}$ ».

Enfin, l'évaluation de l'anecdote sert souvent à souligner son but pragmatique, ce genre de récit ayant fondamentalement une visée perlocutoire. Or, on constate dans de nombreuses occurrences que le narrateur met en abyme l'effet qu'il escompte produire, en figurant un public intradiégétique, miroir du narrataire ${ }^{(61)}$. Concluant 1'histoire d'un mauvais tour joué au courtisan Cavoye par d'autres aristocrates de la cour, Saint-Simon écrit : "L'histoire en fut faite au Roi qui en pensa mourir de rire, et Cavoye et ses familiers de desespoir ${ }^{(62)} \gg$. Ce récit spéculaire met en abyme l'acte narratif et la réaction du roi. Ce dispositif exhibe le processus « de la production et de la réception comme telles ${ }^{(63)}$ ». La chute remplit alors à plein la fonction de cadre interactionnel : le récepteur se sent probablement invité à reproduire les affects qu'il voit représentés intradiégétiquement.

L'étape finale de l'anecdote est donc rarement constituée d'une véritable morale, mais plutôt de commentaires pragmatiques ou axiologiques visant à orienter la réception du récit. Parce que l'enjeu pragmatique est de créer un effet de surprise, on perdrait sans doute à souligner le sens que véhicule l'anecdote par de longues "réflexions », comme on nomme en français classique les commentaires moraux. Ainsi dans son Essai sur le récit de la fin du XVIII ${ }^{\mathrm{e}}$ siècle Bérardier de Bataut écrit-il :

Quand on a de pareilles anecdotes à raconter, il est sage, je crois, de supprimer toute réflexion. Le simple récit en fait naître assez : ce qu'on pourroit ajouter ne feroit que distraire, $\&$ rallentir l'impression naturelle des objets ${ }^{(64)}$.

Pour conclure sur ce point, disons que la $\operatorname{Pn} \Omega$ est facultative dans un genre de récit lapidaire comme l'anecdote, et quand elle est présente, elle est moins un commentaire qu'une zone de transition, souvent dialogique, entre la fin du récit et la suite du texte.

\section{Conclusion}

On a insisté ici sur un ensemble d'indices langagiers qui, rassemblés en faisceaux, constituent l'identité générique de l'anecdote, en particulier son dispositif d'encadrement double : le cadre interne de la séquence ([un jour [...] chute]) et le cadre externe (annonce/évaluation). L'énonciation du cadre externe est le

(60) W. Labov, Le Parler ordinaire, op. cit., p. 410.

(61) Sur l'instance du narrataire et son rôle de relais avec le lecteur, mais aussi de «porte-parole » du narrateur, voir G. Prince, «Introduction à l'étude du narrataire », Poétique, avril 1973, n 14, p. 178-196.

(62) Saint-Simon, Mémoires, op. cit, t. II, p. 297-298.

(63) L. Dällenbach, Le Récit spéculaire : essai sur la mise en abyme, Paris, Éd. du Seuil, coll. «Poétique », 1977, p. 100.

(64) Bérardier de Bataut, Essai sur le récit, ou entretiens sur la manière de raconter, Paris, Ch.P. Berton, 1776, p. 397. 
plus souvent embrayée, car il constitue une zone de passage du discours au récit (et retour). Cette structure concentrique se schématise comme suit :

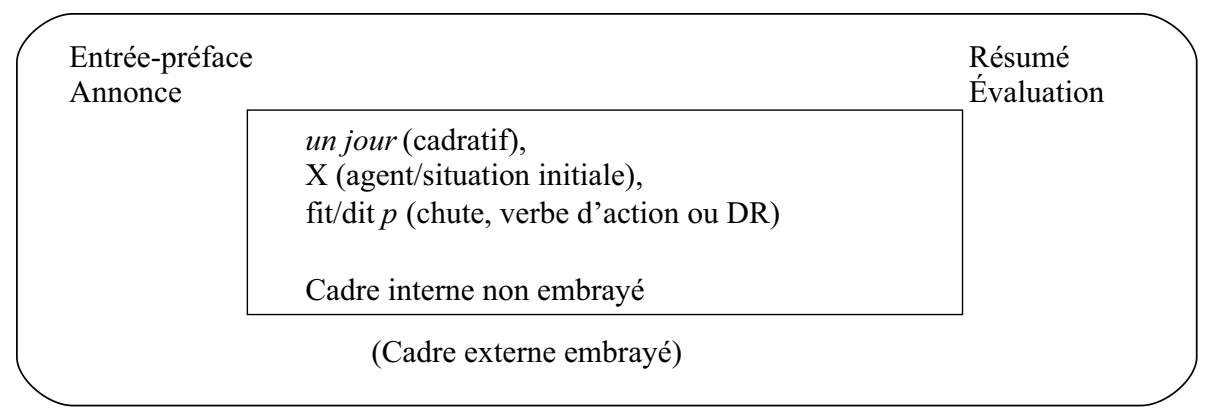

Ces indices laissent penser que l'anecdote apparaît de manière plus ou moins attendue dans le régime des textes (par son cadre marqué, sa cohésion interne caractérisée par la tension vers une chute, son encadrement dialogique). La congruence des faits de langue repérés débouche donc sur l'idée d'une reconnaissance générique dans la perception de la composition séquentielle des longs textes. A. Jaubert a ainsi insisté sur le rôle de la « médiation des genres ${ }^{(65)}$ » dans la cohérence et la cohésion des séquences, en donnant précisément l'exemple de l'anecdote ${ }^{(66)}$. Ces unités sont des « facilitateurs de l'interprétation ${ }^{(67)}$ » : leur repérage permet d'articuler les relations textuelles entre les unités à leurs fonctions et significations.

Il resterait donc à présent à relier ces marqueurs aux rôles que l'anecdote joue dans les textes. Elle est généralement destinée à attirer l'attention d'un destinataire, qu'elle rompe l'abstraction d'un discours théorique, agrémente une explication didactique, ou permette de changer d'échelle dans un récit historique en révélant les petites histoires en marge de la grande. Ce sont toutes des fonctions d'appel du destinataire. Or, pour avoir le relief nécessaire à la réalisation de telles fonctions, l'anecdote se doit d'être nettement découpée sur le fond textuel où elle s'insère : c'est pourquoi ce genre de récit est toujours ostensiblement encadré, et par là toujours visible, si miniature soit-il.

(65) A. Jaubert, «Introduction. Cohésion et cohérence : étapes et relais pour l'interprétation », dans A. Jaubert (dir.), Cohésion et cohérence. Études de linguistique textuelle, op. cit., p. 11.

(66) Ibid., p. 8.

(67) $I d$. 